\title{
Heterotopic ossification with fever of unknown origin
}

\author{
Shigeo Godo MD PhD, Shigeki Kushimoto MD PhD
}

Cite as: CMAJ 2019 February 25;191:E232. doi: 10.1503/cmaj.180617

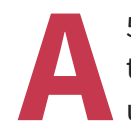

52-year-old man was admitted to hospital with coma from thalamic hemorrhage. He developed a persistent fever of unknown origin during his stay in the intensive care unit (ICU). A tentative diagnosis of central fever was made based on the unremarkable findings of an extensive fever workup. The patient's condition was complicated by prolonged hyperthermia, despite supportive care in the ICU. Repeat computed tomography 4 weeks after presentation showed the development of heterotopic ossification in the bilateral internal obturator muscles (Figure 1). The patient's alkaline phosphatase level rose to a maximum of 4 times the normal range, without elevation of inflammatory and hepatic markers, consistent with a bone-derived elevation. This supported a diagnosis of heterotopic ossification rather than more common explanations for his fever, such as cellulitis, abscess or hematoma. The patient also showed typical features of paroxysmal sympathetic hyperactivity, including episodic paroxysmal tachycardia, hypertension, hyperthermia, tachypnea, diaphoresis and teeth grinding without substantial stressful stimuli. We diagnosed paroxysmal sympathetic hyperactivity, the likely cause of the heterotopic ossification, using recently developed consensus criteria. ${ }^{1}$

Heterotopic ossification is the ectopic formation of mature lamellar bone in nonosseous tissue that usually follows soft tissue trauma and fractures, but also arises secondary to nontraumatic neurologic injury, including paroxysmal sympathetic hyperactivity that can occur following severe acquired brain injury. ${ }^{2}$ Autonomic dysregulation may serve as an osteoinductive factor. ${ }^{3}$ As in our patient, a finding of heterotopic ossification can be a clue to diagnosing paroxysmal sympathetic hyperactivity. ${ }^{4}$ Our patient showed a gradual decline in his paroxysms without specific therapeutic interventions, and the heterotopic ossification remained unchanged.

\section{References}

1. Baguley IJ, Perkes IE, Fernandez-Ortega JF, et al. Paroxysmal sympathetic hyperactivity after acquired brain injury: consensus on conceptual definition, nomenclature, and diagnostic criteria. J Neurotrauma 2014;31:1515-20.

2. Bargellesi S, Cavasin L, Scarponi F, et al. Occurrence and predictive factors of heterotopic ossification in severe acquired brain injured patients during rehabilitation stay: cross-sectional survey. Clin Rehabil 2018;32:255-62.

3. Sakellariou VI, Grigoriou E, Mavrogenis AF, et al. Heterotopic ossification following traumatic brain injury and spinal cord injury: insight into the etiology and pathophysiology. J Musculoskelet Neuronal Interact 2012;12:230-40.

4. Meyfroidt G, Baguley IJ, Menon DK. Paroxysmal sympathetic hyperactivity: the storm after acute brain injury. Lancet Neurol 2017;16:721-9.
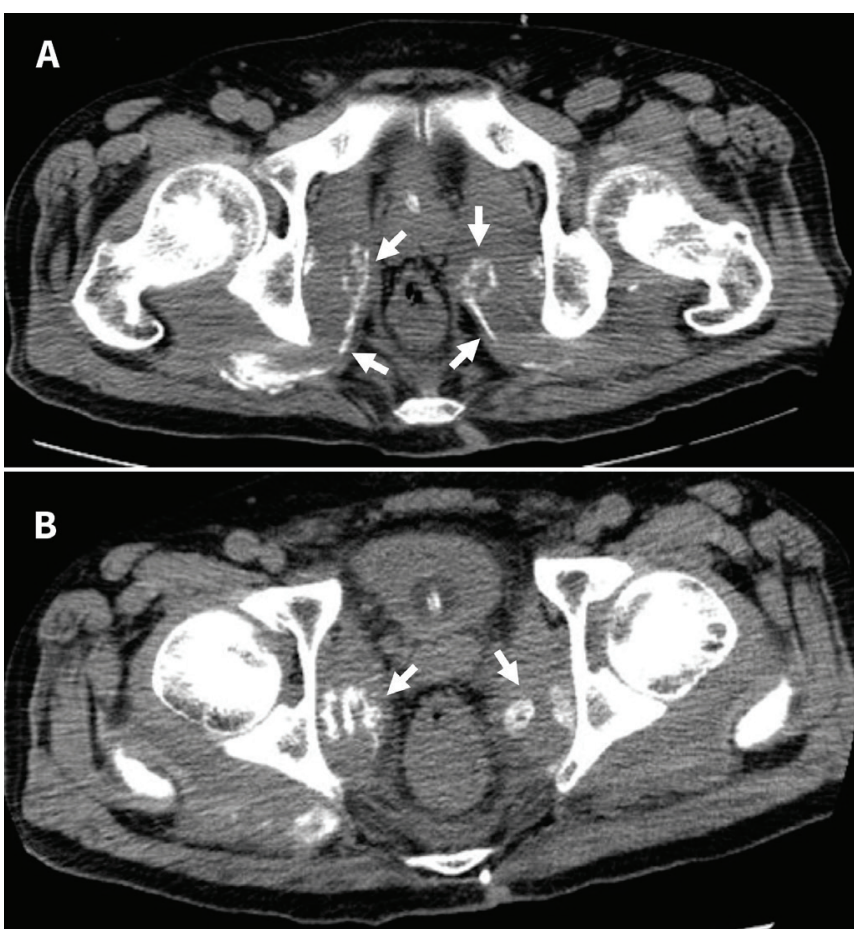

Figure 1: (A, B) Abdominal computed tomographic image in a 52-year-old man with coma from thalamic hemorrhage 4 weeks after presentation, showing heterotopic ossification in the bilateral internal obturator muscles (arrows).

Competing interests: None declared.

This article has been peer reviewed.

The authors have obtained patient consent.

Affiliations: Department of Cardiovascular Medicine (Godo), Tohoku University Graduate School of Medicine; Department of Emergency and Critical Care Medicine (Godo, Kushimoto), Tohoku University Hospital; Division of Emergency and Critical Care Medicine (Kushimoto), Tohoku University Graduate School of Medicine, Sendai, Japan

Correspondence to: Shigeo Godo, s-godo@cardio.med.tohoku.ac.jp 